\title{
Pneumatoceles in Hyperimmunoglobulin E syndrome: Is screening warranted?
}

\author{
Manouri P Senanayake ${ }^{1}$, P Irantha Karunaratne ${ }^{2}$, Leslie Gunarathna ${ }^{3}$
}

Sri Lanka Journal of Child Health, 2015; 44(1): 65-66

(Key words: Hyperimmunoglobulin E syndrome; pneumatoceles; screening for complications)

\section{Introduction}

Hyper-immunoglobulin E syndrome (HIES) is a rare primary immunodeficiency affecting both boys and girls ${ }^{1}$. Characteristic clinical features include recurrent skin abscesses, sino-pulmonary infections, chronic dermatitis, abnormal facial features, skeletal abnormalities and/or dental abnormalities ${ }^{1}$. A serum immunoglobulin E (IgE) level exceeding $2000 \mathrm{IU} / \mathrm{ml}$ is considered diagnostic ${ }^{1}$. Staphylococcal bronchopneumonia, lung abscess, bronchiectasis and single or multiple pneumatoceles are known serious pulmonary complications of $\mathrm{HIES}^{2}$. We present a child with HIES, who was detected to have a large pneumatocele as an incidental finding, despite not having had past or present lung infection,

\section{Case report}

A nine year old school girl from a farming community in rural Sri Lanka was referred by the local hospital for recurrent skin abscesses from the age of 6 months. Apart from suppurative otitis media there had been no other major infections. Respiratory infections or contact with tuberculosis were absent. There was no consanguinity. Her parents and younger brother were healthy. Physical examination found a systemically well and afebrile child of average build. She had a large, non tender, fluctuant lump in the right mandibular region (Figure 1). A bulbous nasal tip, two rows of teeth in lower jaw, eczematous rash with pitted scarring and multiple healed surgical scars over scalp, face and legs, were present (Figure 2).

${ }^{1}$ Senior Professor in Paediatrics, Faculty of Medicine, University of Colombo, ${ }^{2}$ Senior Registrar in Paediatrics, ${ }^{3}$ Registrar in Paediatrics, Professorial Paediatrics Unit, Lady Ridgeway Hospital, Colombo

(Received on 28 January 2014: Accepted after revision on 14 March 2014)

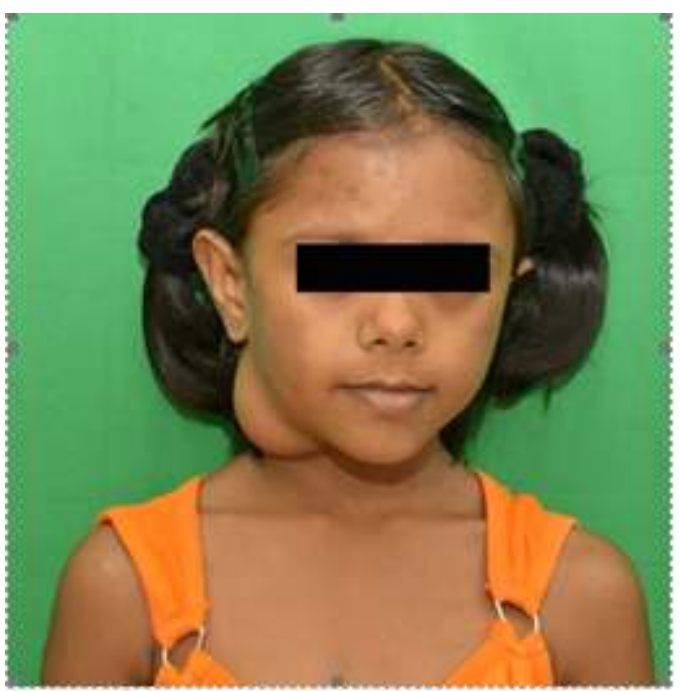

Figurel: Large abscess in left cervical region

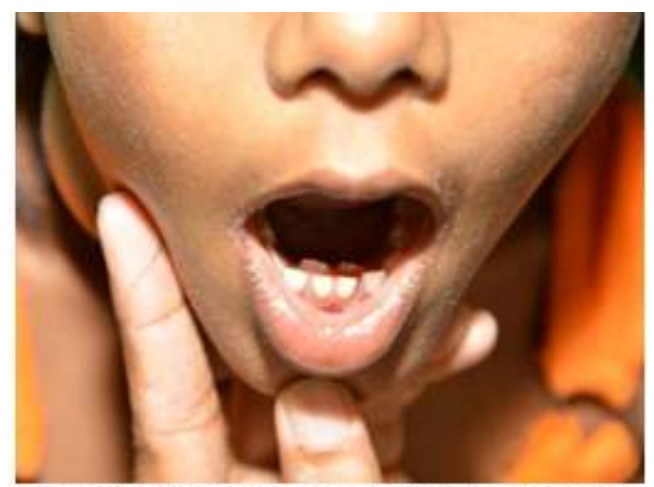

Figure 2: Two rows of teeth in lower dentition and bulbous tip of nose

There was no cough, tachypnoea, clubbing or crackles on auscultation. Rest of systems examination was normal. Blood investigations found haemoglobin $9.3 \mathrm{~g} / \mathrm{dl}$, white blood count $10.4 \times 10^{3}$ (N-54, L-39 and E-5), hypochromic microcytic red cells and normal white cell morphology on blood picture, erythrocyte sedimentation rate $20 \mathrm{~mm}\left(1^{\text {st }}\right.$ hour) and serum IgE level over $2000 \mathrm{IU} / \mathrm{ml}$. Chest 
radiograph showed a large pneumatocele in the right upper lung (Figure 3). Two rows of teeth were shown in radiograph of mandible (Figure 4)

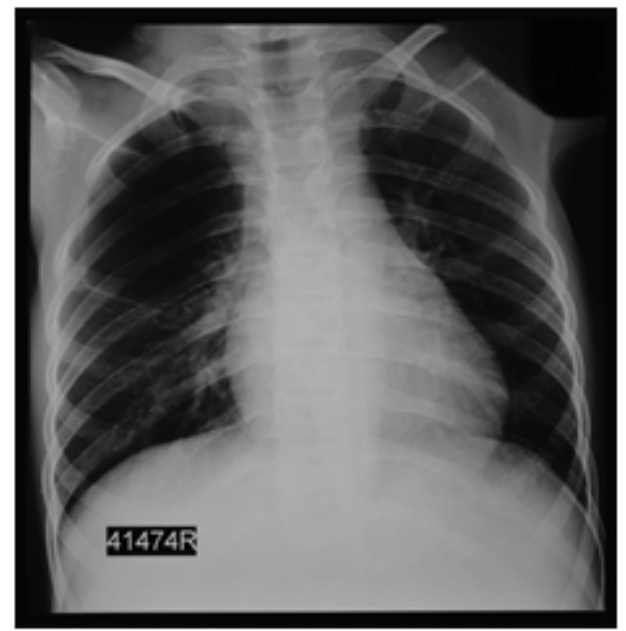

Figure 3: A large pneumatocele in upper lobe of right lung

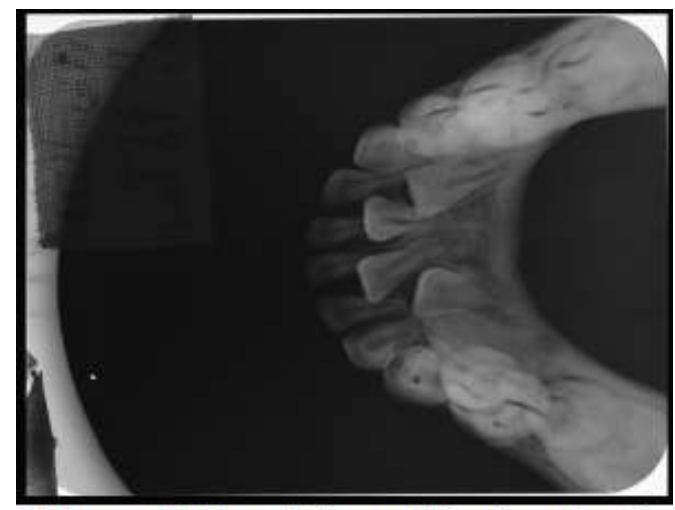

Figure 4: X-ray of mandible showing 2 rows of teeth

Mediastinum and heart shadow were normal. Excision biopsy found an enlarged lymph node containing pus which grew a pure growth of Staphylococcus aureus on culture. Histology showed scanty neutrophil infiltration of abscess wall without caseation.

\section{Discussion}

Hyperimmunoglobulin E syndrome (hyper-IgE, Job syndrome, HIES) was diagnosed based on characteristic clinical features (Figures 1 and 2) and markedly elevated serum IgE level of over 2000 $\mathrm{IU} / \mathrm{ml}$ which is the hallmark value of diagnostic significance. In keeping with the original description of this syndrome, named after the biblical character Job (who was afflicted with numerous "sores and boils") the prominent clinical feature in this girl was recurrent skin abscesses. Retained primary teeth are described in $70 \%$ of reported cases with the syndrome . $^{3}$.

Reduced neutrophil chemotaxis that is described in this condition explains the 'cold' nature of abscesses, lack of acute inflammatory features and the histologic finding of scanty neutrophil infiltration ${ }^{4}$. Staphylococcal chest infections can result in asymptomatic pneumatoceles and our patient did not have any significant respiratory infection to account for the large asymptomatic pneumatocele detected on radiography.

Pneumatoceles in HIES are known to persist and even have a relentless natural history of recurrent infections necessitating surgical resection. We wish to highlight the need to screen patients with HIES for this pulmonary complication even when there are no antecedent or current respiratory infections.

\section{References}

1. Erlewyn-Lajeunesse MD: Hyperimmunoglobulin E syndrome with recurrent infection: a review current opinion and treatment. Pediatric Allergy Immunology 2004; 11:133-41.

http://dx.doi.org/10.1034/j.13993038.2000.0009 $\underline{1 . \mathrm{X}}$

2. Shamberger RC, Wohl ME, Perez-Atayde A, Hendren WH. Pneumatocele complicating Hyperimmunoglobulin E syndrome (Job's Syndrome). The Annals of Thoracic Surgery 1992; 54(6):1206-8. http://dx.doi.org/10.1016/0003-4975(92)90100-I

3. Freeman AF, Holland SM. Clinical manifestations, aetiology, and pathogenesis of the Hyper-Ig E syndrome. Paediatrics. 2009; 65:32R-37R.

http://dx.doi.org/10.1203/PDR.0b013e31819dc8 c5

4. Paulson ML, Freeman AF, Steven M. Holland SM. Hyper IgE Syndrome: An update on clinical aspects and the role of Signal Transducer and Activator of Transcription. Current Opinion in Allergy and Clinical Immunology 2008; 8(6):527-33. Available on: http://www.medscape.com/viewarticle/584253 4 